\title{
Die Umgestaltung des glazigenen Reliefs der norddeutschen Altmoränengebiete am Beispiel des Uelzener Beckens ${ }^{1)}$
}

\author{
Von Jürgen Hagedorn, Göttingen
}

Mit 3 Abbildungen im Text

$\mathrm{Zusammenfassung.} \mathrm{Der} \mathrm{Einfluß} \mathrm{der} \mathrm{weicheleiszeitlichen} \mathrm{periglazialen} \mathrm{Formung} \mathrm{auf}$ die Umgestaltung der Altmoränengebiete wird am Beispiel des im Norddeutschen Tiefland gelegenen Uelzener Beckens untersucht. Dieses Gebiet erhielt seine grundlegende Formung durch das Warthe-Inlandeis und lag während der Weichsel-Eiszeit im periglazialen Bereich.

Es werden zwei Beispiele für eine durch fluviale und solifluidale Prozesse verursachte Reliefumkehr im kleinen gezeigt. Eine Abschätzung der Größenordnung der Abtragung im Beckenbereich 1.) seit dem Rückzug des Warthe-Eises und 2.) seit dem Eem-Interglazial wird ermöglicht 1.) durch die Umkehr der Entwässerung nach dem Eiszerfall und 2.) durch die Höhenlage Eemzeitlicher Ablagerungen über dem heutigen Beckenboden. Danach muß man mit einer allgemeinen Erniedrigung des Beckenbodens um etwa $20 \mathrm{~m}$ seit der ausgehenden Warthe-Zeit rechnen; davon sind etwa $10 \mathrm{~m}$ allein der weichseleiszeitlichen Abtragung zuzuschreiben.

$\mathrm{S} u \mathrm{~mm}$ a $\mathrm{r}$. The study deals with the influence of the Weichselian periglacial shaping on the modification of older moraine areas; the basin of Uelzen, which is part of the North German lowland, has been taken as an example. It was by the inland-ice of the Warthe-period that this area was fundamentally formed, and during the Weichselian period it was subject to periglacial processes.

Two examples of a small-scale relief inversion caused by fluvial and soliflual processes are demonstrated. A calculation of the dimension of denudation in the basin itself, which has taken place 1.) since the retreat of the Warthe-glaciers and 2.) since the Eem interglacial stage, is possible; it is based 1.) upon the inversion of the drainage after the shrinkage of the ice and 2.) on the altitude of Eem interglacial sediments above the present basin bottom. According to these data an average degradation of the basin bottom of about 20 metres since the late Warthe period must be assumed; about 10 metres of this account are due to the Weichselian denudation exclusively.

Seitdem sich - vor allem als Ergebnis der Arbeiten Gripps (1924, 1925 u. a.) - die Erkenntnis durchgesetzt hat, daß die weichseleiszeitlichen periglazialen Formungsprozesse wesentliche Reliefunterschiede zwischen Jung- und Altmoränengebieten hervorgerufen haben, hat sich die Forschung um eine genauere Kenntnis des Ausmaßes der weichseleiszeitlichen Abtragung und Formung in den Altmoränengebieten bemüht. V. MiLthers (in Jessen \& Milthers 1928), Gripp (1938) und Dewers (1934, 1950) sprachen sich dabei für eine erhebliche weichseleiszeitliche Abtragung aus und leiteten Abtragungsbeträge von $10 \mathrm{~m}$ und mehr ab; aber Dewers selbst schränkte in einer anderen Arbeit (1941) die Abtragungsleistung in den norddeutschen Periglazialgebieten der Weichseleiszeit wieder wesentlich ein. Von den jüngeren Forschungen lassen die Untersuchungen PICARds (1959) in Westholstein eher auf eine große Bedeutung der periglazialen Abtragung für die Umgestaltung des Altmoränenreliefs schließen, während ILLIEs (1955) und H. HAgedorN (1961) den periglazialen Prozessen nur eine unbedeutende Überformung des älteren Reliefs zuschreiben möchten. Aufgrund von Untersuchungen im Uelzener Becken (J. HAGEDORN 1964) glaube ich zu dieser Frage einige neue Hinweise beitragen zu können.

Das Uelzener Becken ist ein Teil der nordöstlichen Lüneburger Heide. Ein im Mittel $50 \mathrm{~m}$ ü. NN gelegener flacher Beckenboden, in den die Täler der Ilmenau und ihrer Nebenflüsse nur wenig eingetieft sind, wird allseitig von bis zu $130 \mathrm{~m}$ ü. NN ansteigenden Höhen umrandet. Diese Beckenumrandung wird im W, S und E durch gleichaltrige warthezeitliche Stauchmoränen gebildet, die sich im W und SW an die äußerste Randlage

1) Vortrag, gehalten auf der Hauptversammlung der Deutschen Quartärvereinigung am 10. Oktober 1964 in Lüneburg. 


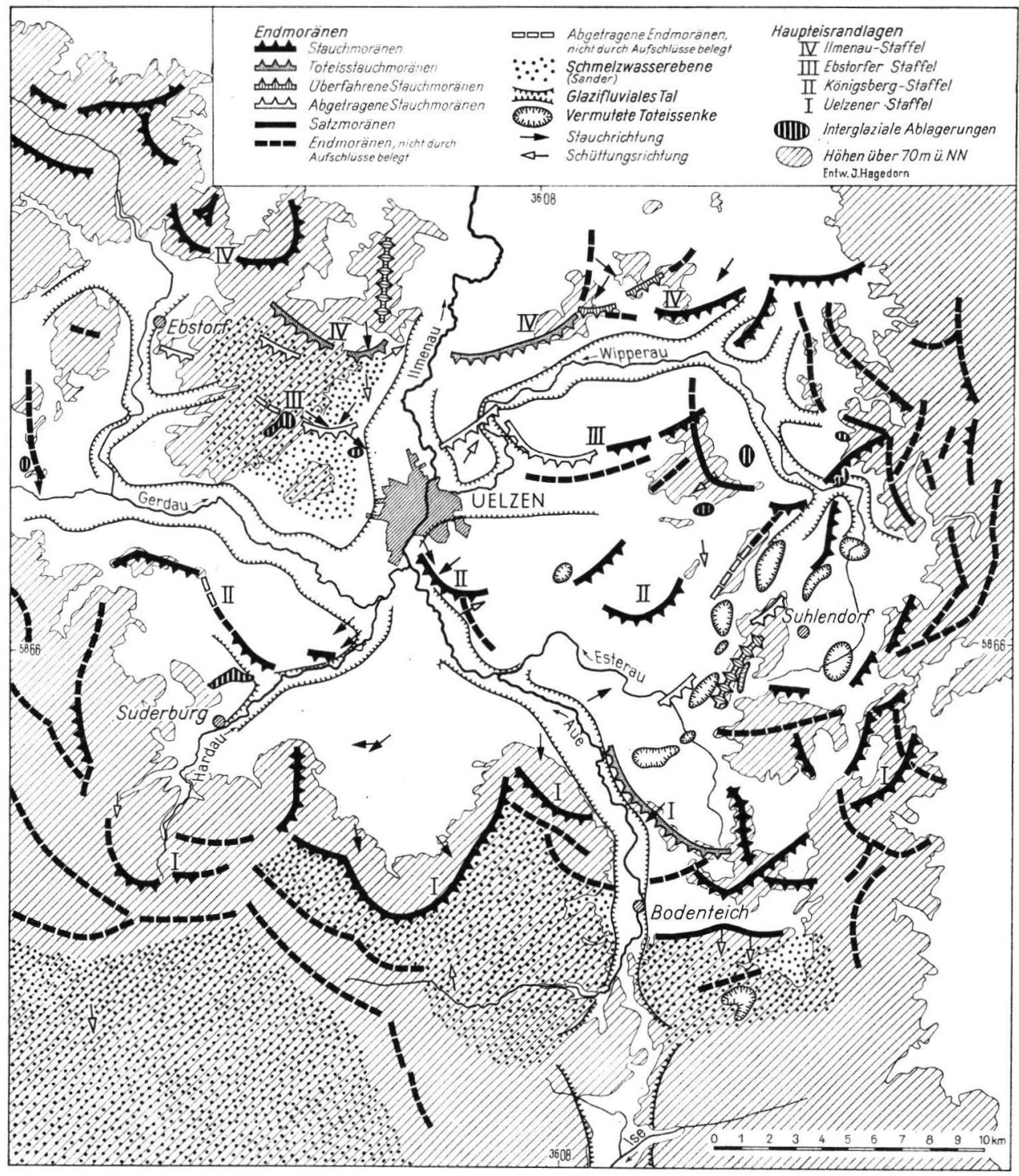

Abb. 1. Glazialmorphologische Übersichtskarte des Uelzener Beckens.

des Warthe-Eises anlehnen (vgl. Abb. 1). Im N wird das Becken durch die Stauchmoränen eines jüngeren warthezeitlichen Gletschervorstoßes abgeriegelt. Endmoränenreste untergeordneter Eisrandlagen befinden sich im Inneren des Beckens, das in groben Zügen die Umrisse eines ehemaligen Zungenbeckens wiedergibt. Sanderflächen, die einigen Endmoränen vorgelagert sind, und die in den größeren Talungen erkennbaren glazifluvialen Täler kennzeichnen die Entwässerung der Eisrandlagen der Warthezeit.

Die Zeugnisse der weichseleiszeitlichen Abtragung und Formung sind uns am deutlichsten in der Vielzahl der Dellen und Flachmuldentäler in den Gebieten flacheren Reliefs vor allem des Beckeninnern und in dem dichten Netz steilhängiger Trockentäler an den steileren Abdachungen vor allem der Beckenumrandung überliefert. Einzelbeobachtungen 
lassen erkennen, daß die Umgestaltung des Reliefs während der Weichseleiszeit über die Einschneidung dieser Täler durchaus hinausging. Das sei an zwei Beispielen gezeigt.

Den Endmoränen der südlichen Beckenumrahmung ist der bereits von WoLDSTEDT (1939) genannte Bokeler Sander vorgelagert. Daraus erhebt sich im E, am Rande der nach WoLDSTEDT durch Toteisschmelzen entstandenen Senke von Reinstorf, die Kuppe des Fahrbergs. Die Bearbeiter der Geologischen Ubersichtskarte von Nordwestdeutschland (1951) sahen in ihm noch den Teil einer Endmoräne. Ein Vergleich der Höhenlage der Kuppe mit der des Sanderansatzes an den Endmoränen und Aufschlüsse auf der Kuppe zeigen aber, daß hier zweifellos ein Teil des ehemaligen Sanders vorliegt. Die Kuppe wird aus ungestörten feinsandigen bis tonigen Sedimenten aufgebaut (Abb. 2), so daß man nach Korngröße und Art der Lagerung sogar auf einen ehemaligen Senkenbereich in der Sanderfläche schließen kann. Man könnte hier also von einer Reliefumkehr im kleinen sprechen.

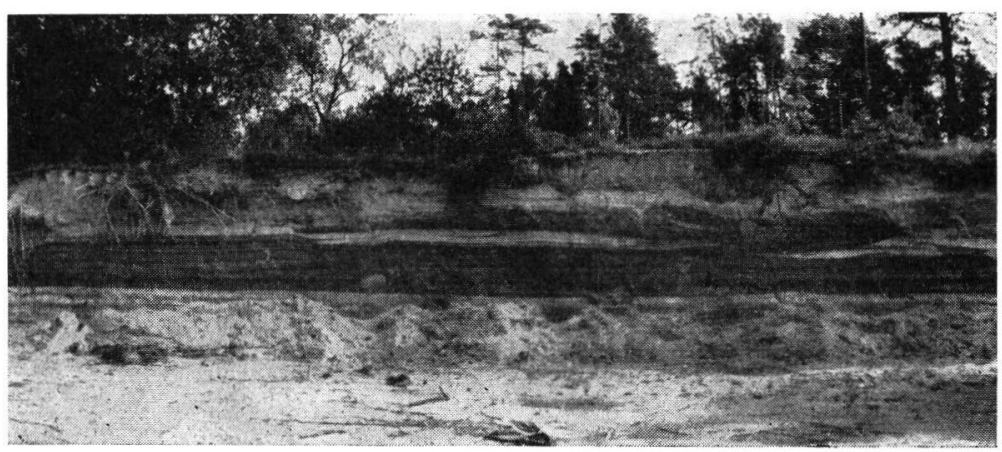

Abb. 2. Beckensedimente in ungestörter Lagerung auf der Kuppe des Fahrberges, westlich Bodenteich.

Diese Reliefumkehr ist nicht allein dem Toteis zuzuschreiben. Es läßt sich vielmehr erkennen, daß fluviale Täler den Fahrberg im N und S aus der ehemaligen Sanderfläche herausgeschnitten haben. Diese Täler werden heute kaum durchflossen. Ihrer Form nach unterscheiden sie sich nicht von anderen trockenen Tälern im Uelzener Becken und seiner Umrandung und müssen wie diese als im wesentlichen unter den periglazialen Bedingungen der Weichseleiszeit entstanden angesehen werden.

Eine andere Beobachtung erhellt die von den Dellen ausgehende Abtragungs- und Formungswirkung: Nördlich von Hamerstorf war in einer Kiesgrube der Querschnitt einer ehemaligen Delle zu erkennen, die mit Abtragungsschutt verfüllt und nicht reaktiviert worden ist (Abb. 3). Vielmehr entstanden durch Eintiefung an anderer Stelle neue Dellen, so daß sich über der verschütteten Delle fast schon eine Reliefumkehr anbahnt.

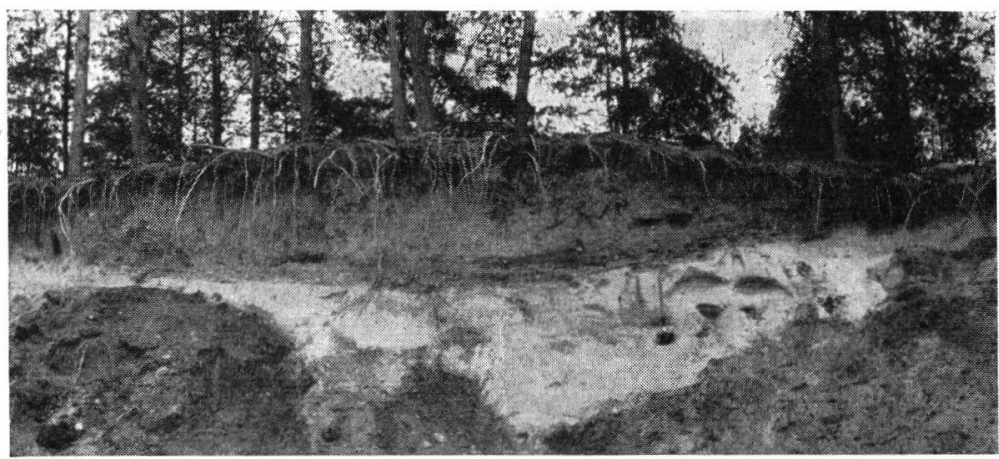

Abb. 3. Anfänge einer Reliefumkehr über schutterfüllter ehemaliger Delle, nördlich Hamerstorf. 
Durch solche Verlegung von Dellen muß der flächenhafte Charakter der periglazialen Abtragung, der in den im Beckeninnern überlieferten flachen Hohlformen erkennbar ist, noch verstärkt worden sein. Das verdient Beachtung, wenn wir uns an eine Abschätzung des Betrages der Abtragung im Uelzener Becken heranwagen.

Bei dieser Abschätzung kann man davon ausgehen, daß die heute zur Elbe gerichtete Entwässerung des Uelzener Beckens ursprünglich über das heutige Isetal und das Oertzetal nach $S$ zum Allerurstromtal erfolgte, was sich im Relief deutlich widerspiegelt (vgl. dazu die Höhenschichtenkarte der Lüneburger Heide von OlвRicht 1910). Dieser Abfluß muß mindestens solange bestanden haben, wie die Entwässerung nach $\mathrm{N}$ durch das warthezeitliche Inlandeis plombiert war. $\mathrm{Da}$ im Uelzener Becken nicht mehr überfahrene Ablagerungen eines Stausees fast völlig fehlen, kann man nicht auf einen nach dem Eisrückgang vorhandenen Zungenbeckensee mit einem Überlauf an der niedrigsten Stelle seines Randes schließen, sondern muß annehmen, daß das Ilmenautal mit seinen Nebentälern ursprünglich das Oberlaufsgebiet des Isetales und z. T. des Oertzetales im Uelzener Becken darstellte. Diese Annahme wird dadurch gestützt, daß die Täler aller größeren Ilmenau-Nebenflüsse einen etwas nach $S$ gerichteten Verlauf zeigen, an der Mündung aber nach $\mathrm{N}$ verschleppt werden. Die Ise wurde demnach durch die von der wesentlich tiefer als das Allertal gelegenen Erosionsbasis des Elbetales rückschreitende Erosion ihres Oberlaufgebietes beraubt.

Die Talwasserscheiden zwischen Ilmenau und Ise bzw. zwischen Gerdau und Oertze liegen heute bei $70 \mathrm{~m}$ ü. NN. Da eine Aufschotterung im Wasserscheidenbereich nach der Gefällsumkehr nicht anzunehmen ist und auch nicht mit einer tektonischen Absenkung des Uelzener Beckens gerechnet werden kann, muß man schließen, daß das ehemalige Oberlaufgebiet der nach S entwässernden Flüsse im Uelzener Becken mindestens in Höhe der heutigen Wasserscheide gelegen hat, d. h. ebenfalls $70 \mathrm{~m}$ ï. NN. Heute liegt der Beckenboden im Mittel $50 \mathrm{~m}$ ü. NN. Daraus ergibt sich eine Abtragung seit der Gefällsumkehr von etwa $20 \mathrm{~m}$, und zwar weitgehend flächenhaft.

Auf die Gefällsumkehr der Ilmenau hat bereits ILLIES (1952) aufgrund von Untersuchungen im Unterelbegebiet hingewiesen. Er stellt sie zeitlich für den Bereich des Uelzener Beckens in das Weichsel-Hochglazial (1952, S. 551). Die Verhältnisse im Uelzener Becken bestätigen diese Datierung nicht. U. a. liegen hier alle bekannten eemzeitlichen Ablagerungen, die nur randlich erhalten sind (vgl. Abb. 1), etwa $60 \mathrm{~m}$ ü. NN. Ungefähr soweit muß also der Beckenboden bereits bis zur Eemzeit abgetragen worden sein. Das ist aber nach der oben gegebenen Ableitung wiederum nur nach einer vorher erfolgten Gefällsumkehr denkbar. Diese dürfte daher in die Zeit unmittelbar nach dem Rückzug des Wartheeises bis zum Elbetal fallen. In der ausgehenden Warthezeit wurde demnach der Beckenboden bereits um etwa $10 \mathrm{~m}$ erniedrigt. Es bleibt dann noch ein Abtragungsbetrag von weiteren $10 \mathrm{~m}$, den wir im wesentlichen den weichseleiszeitlichen Vorgängen zuschreiben müssen; denn die Abtragung im Holozän kann bei der ohnehin gegebenen Ungenauigkeit der Abschätzung vernachlässigt werden.

Dieser sehr erhebliche Betrag der weichseleiszeitlichen periglazialen Abtragung könnte fast unmöglich erscheinen, wenn man an manche neueren Beobachtungen aus den rezenten Periglazialgebieten denkt, die eher eine Reduzierung der bisherigen Schätzungen über die periglaziale Abtragung in den nicht vereisten Gebieten Mitteleuropas nahelegen, wie z. B. die Untersuchungen von KLimaszewski (1960) auf Spitzbergen. Aber es ist zu beachten, daß die Altmoränengebiete aus Lockergestein aufgebaut sind, aus Material also, das, ohne der Aufbereitung durch die Verwitterung zu bedürfen, nach dem sommerlichen Auftauen den periglazialen Abtragungsprozessen leichte Arbeit gab. Auch ist im speziellen Falle des Uelzener Beckens wahrscheinlich zu berücksichtigen, daß durch die Gefällserhöhung nach der jungen fluvialen Anzapfung hier besonders günstige Bedingungen für den fluvialen Abtransport des durch Solifluktion und flächenhaft wirkende Abspülung den größeren 
Tälern zugeführten Materials gegeben waren. Der heutige Beckenboden erweist sich also als eine Abtragungsfläche. Damit wird zugleich erklärt, warum diese Fläche sehr unterschiedliches glazigenes Material überspannt. Auch ehemalige Endmoränen sind fast völlig in diese Fläche einbezogen worden, z. T. allerdings durch glazifluviale Abtragung, und nur geologische Aufschlüsse ermöglichen uns stellenweise noch den Schluß auf ehemalige Vollformen, die durch Eisvorstöße geschaffen wurden. Da mit ähnlichen Verhältnissen auch in anderen Teilen des norddeutschen Tieflandes gerechnet werden muß, ist es notwendig, die mögliche weichseleiszeitliche Abtragung bei Versuchen einer Feingliederung der Warthezeit und älterer Eiszeiten mit Hilfe der Verknüpfung von Eisrandlagen als bedeutenden Unsicherheitsfaktor zu berücksichtigen.

\section{Zitierte Literatur:}

Dewers, F.: Einige wesentliche Charakterzüge der nordwestdeutschen Diluvialmorphologie. - Abh. naturw. Ver. Bremen 29, 33-47, Bremen 1934. - - Das Diluvium. In: Das Känozoikum in Niedersachsen. - Geologie und Lagerstätten Niedersachsens 3 - Schr. Wirtschaftswiss. Ges. z. Studium Nieders. NF 3, S. 53-267, Oldenburg 1941. - - Einige charakteristische Züge der Oberflächengestalt des norddeutschen Flachlandes. - N. Archiv f. Niedersachsen, 18, 475-488, Bremen-Horn 195,0.

GriPP, K.: Über die äußerste Grenze der letzten Vereisung in Nordwestdeutschland. - Mitt. geogr. Ges. Hamburg 36, 159-245, Hamburg 1924. - - Uber eine morphologische Grenze im nordwestdeutschen Flachlande und deren Bedeutung. - Z. deutsch. geol. Ges. 77, Monatsberichte, 128-130, Berlin 1925. - - Der Oberflächenabtrag im Alt-Diluvium und seine Bedeutung für das Vorkommen paläolithischer Funde. - Offa 4, 59-82, Neumünster 1939.

Hagedorn, H.: Morphologische Studien in den Geestgebieten zwischen Unterelbe und Unterweser. - Göttinger geogr. Abh. 26, Göttingen 1961.

Hagedonn, J.: Geomorphologie des Uelzener Beckens. - Göttinger geogr. Abh. 31, Göttingen 1964. IlliEs, H.: Die eiszeitliche Fluß- und Formengeschichte des Unterelbe-Gebietes. - Geol. Jb. 66, 525-5,58, Hannover 1952. - - Die Vereisungsgrenzen in der weiteren Umgebung Hamburgs, ihre Kartierung und stratigraphische Bewertung. - Mitt. geogr. Ges. Hamburg 51, 7-54, Hamburg 1955.

Klimaszewski, M.: Geomorphological Studies of the Western Part of Spitzbergen between Kongsfjord and Eidembukta. - Zeszyty Naukowe Univ. Jagiellônskiego 32, Prace Geograficzne Z. I, 93-167, Krakow 1960.

Jessen, K. \& Milthers, V.: Stratigraphical and Palaeontological Studies of Interglacial FreshWater Deposits in Jutland and Northwest Germany. - Danmarks geol. Unders., II. R., 48, Kobenhavn 1928.

PiCARD, K.: Gliederung pleistozäner Ablagerungen mit fossilen Böden bei Husum/Nordsee. N. Jb. Geol. Paläont., Monatshefte 6, 259-272, Stuttgart 1959.

Woldstedt, P.: Die Endmoränen in der südlichen Lüneburger Heide. - Abh. naturw. Ver. Bremen 31, 236-246, Bremen 1939.

Karten :

Olbricht, K.: Höhenschichtenkarte der Lüneburger Heide $1: 200000$. - Petermanns Geogr. Mitt. 56, Gotha 1910.

Bentz, A. (Herausg.): Geologische Ubersichtskarte von Nordwestdeutschland $1: 300000$. - Hannover 1951.

Manuskr. eingeg. 2. 3. 1965.

Anschrift des Verf.: Dr. J. Hagedorn, 34 Göttingen, Herzberger Landstr. 2, Geographisches Institut der Universität. 